\title{
Mesosphere and lower thermosphere temperature anomalies during the 2002 Antarctic stratospheric warming event
}

\author{
S. M. I. Azeem ${ }^{1,2}$, E. R. Talaat ${ }^{3}$, G. G. Sivjee ${ }^{2}$, and J.-H. Yee $^{3}$ \\ ${ }^{1}$ ASTRA LLC., San Antonio, TX, USA \\ ${ }^{2}$ Embry-Riddle Aeronautical University, Daytona Beach, FL, USA \\ ${ }^{3}$ Applied Physics Laboratory, Johns Hopkins University, Laurel, MD, USA
}

Received: 4 November 2009 - Revised: 12 January 2010 - Accepted: 15 January 2010 - Published: 25 January 2010

\begin{abstract}
We present kinetic temperatures at $\sim 87 \mathrm{~km}$ and $\sim 94 \mathrm{~km}$ altitudes inferred from $\mathrm{OH}(6,2)$ and $\mathrm{O}_{2} \mathrm{At}(0,1)$ airglow observations, respectively, at South Pole $\left(90^{\circ} \mathrm{S}\right)$, Antarctica in the austral winter of 2002. These $\mathrm{OH}$ and $\mathrm{O}_{2}$ rotational temperatures measurements show mesosphere and lower thermosphere (MLT) temperature anomalies prior to the 2002 Southern Hemisphere Sudden Stratospheric Warming (SSW). In this paper we focus on the first of the three minor stratospheric warmings which preceded the major SSW event. Temperature anomalies observed in the MLT region show sudden cooling $(\Delta T=\sim 30 \mathrm{~K})$ in $\mathrm{OH}$ temperatures accompanied by warming $(\Delta T=\sim 15 \mathrm{~K})$ in $\mathrm{O}_{2}$ temperatures preceding the onset of SSW event by about three to four weeks. This shallow vertical extent of mesospheric cooling is in agreement with the numerical simulation of Coy et al. (2005), however, the model cooling was centered well below the mesopause level. The other observed feature of the South Pole MLT temperature dataset is the intensification of planetary wave activity prior to the onset of SSW event. Fourier analyses of both $\mathrm{OH}$ and $\mathrm{O}_{2}$ temperatures show amplification of planetary wave activity in the 5-10 day range prior to the onset of SSW. The timing of wave amplification seen in the wave spectra coincides with the peak in $\mathrm{OH}$ and $\mathrm{O}_{2}$ temperature anomalies.
\end{abstract}

Keywords. Atmospheric composition and structure (Airglow and aurora) - Meteorology and atmospheric dynamics (Middle atmosphere dynamics; Polar meteorology)

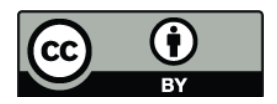

Correspondence to: S. M. I. Azeem (iazeem@astraspace.net)

\section{Introduction}

The winter polar stratosphere is characterized by an extensive vortex region that dynamically interacts with the winter mean flow. Under certain atmospheric conditions, this polar vortex is abruptly disrupted and the mean winds in the high latitude winter stratosphere reverse from westerly to easterly, causing Sudden Stratospheric Warmings (SSW) (Scherhag, 1960; Labitzke, 1965). Such an event can strongly affect the entire middle atmosphere, causing variations in the mesosphere and lower thermosphere (MLT) region circulation (Lysenko et al., 1975; Lauter and Schminder, 1976). During a "major" SSW event, the polar stratosphere warms up by $\sim 60 \mathrm{~K}$ (Schoeberl, 1978) within a space of few days and the net zonal mean wind flow becomes easterly at $60^{\circ}$ at $32 \mathrm{~km}$ height or below (Labitzke and Naujokat, 2000). In contrast, a "minor" SSW event occurs when the polar temperature increases more than $25 \mathrm{~K}$ in a period of a week or less at any stratospheric level without a reversal of zonal-mean circulation.

Recent studies of thermodynamic anomalies in the MLT region associated with the SSW events have confirmed earlier suggestions of stratospheric disturbances influencing the dynamics of the middle and upper atmosphere. Azeem et al. (2005) analyzed the 2002 austral winter temperature data at $\sim 87 \mathrm{~km}$, inferred from South Pole $\mathrm{OH}(3,1)$ airglow measurements, and showed that the strong 4-day wave signature seen in the temperature data was associated with the SW event. Radar studies of Espy et al. (2005) and Pancheva et al. (2008) also have showed similar intensification of planetary waves prior to the onset of the SSW event. Planetary wave amplification in the MLT region prior to the SSW event was also confirmed by radar wind measurements from Antarctica (Mbatha et al., 2009; Dowdy et al., 2007). Numerical simulations of Coy et al. (2005) and Liu and Roble (2002) of the 2002 Southern SSW event showed increased wave 1 amplitude in the MLT region associated with the

Published by Copernicus Publications on behalf of the European Geosciences Union. 

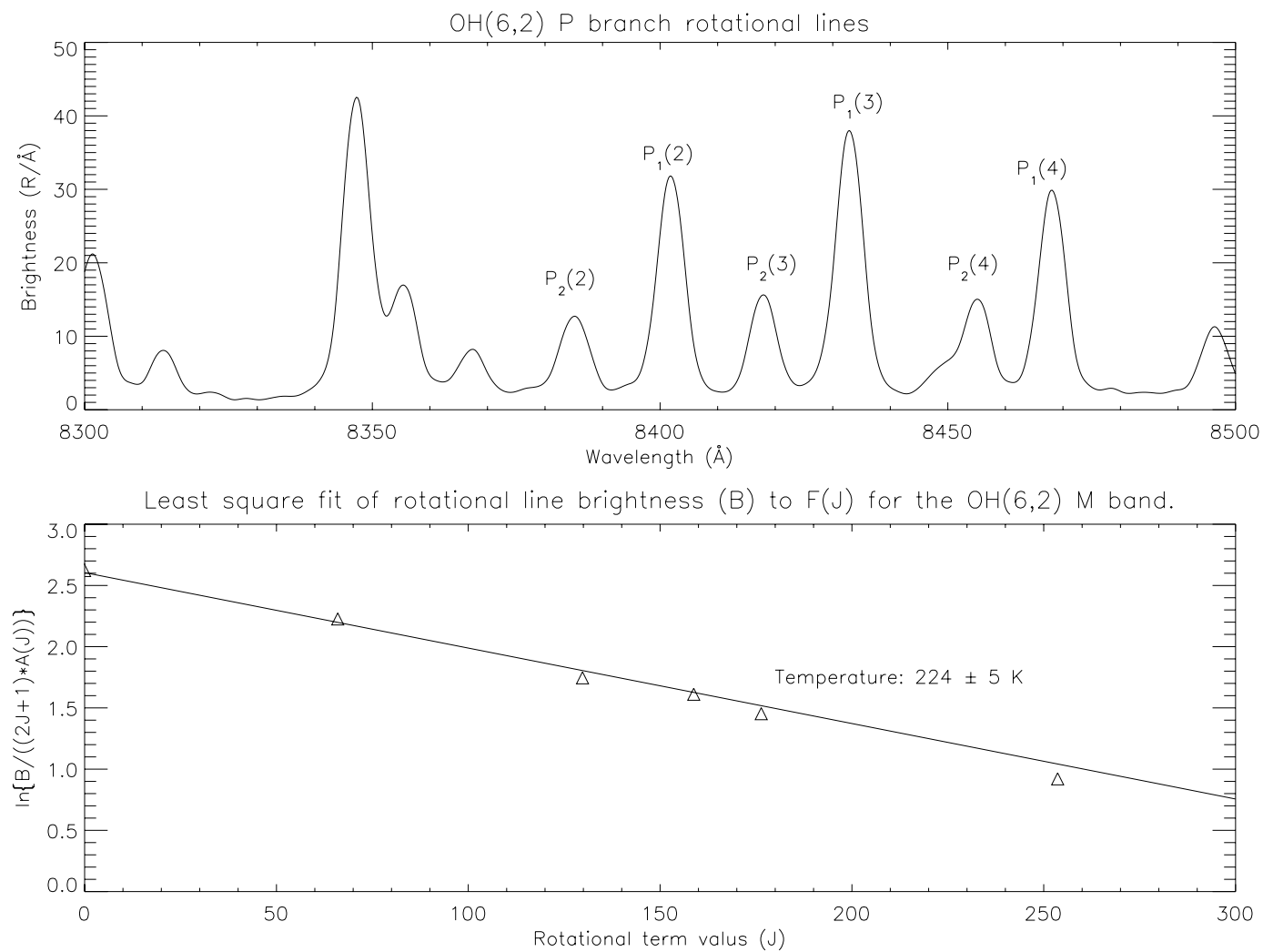

Fig. 1. Top panel: Rotational lines from the $\mathrm{P}$ branch of the airglow $\mathrm{OH}(6,2)$ band recorded by the South Pole CCD spectrograph. Bottom panel: Linearized plot of the first six rotational lines of $\mathrm{OH}(6,2) \mathrm{P}$ branch shown as a function of $F(J)$ and the linear least squares fit to the peak brightness of the rotational lines from which the temperature is calculated.

occurrence of the SSW. It is now well recognized that sudden cooling in mesospheric temperatures are associated with SSW events (Walterscheid et al., 2000; Hernandez, 2003; Cho et al., 2004; Mukhtarov et al., 2007). However, this association between SSW events and the MLT region is not fully understood. For example, the timing of MLT coolings and SSW events remains an open question with Myrab $\varnothing$ et al. (1984) reporting a 1-2 day delay in the mesospheric response to the SSW and Hernandez (2004) suggesting a mesospheric cooling episode leading the SSW by up to 2 months. Furthermore, zonal winds observed by Medium Frequency (MF) and Super Dual Auroral Radar Network (SuperDARN) radars in Antarctica showed wind reversals in the MLT region preceding the wind reversal in the stratosphere by 4 to 7 days (Dowdy et al., 2004; Mbatha et al., 2009). The depth of mesospheric cooling associated with the SSW events is also not yet well established with a wide range of vertical extents being reported in the literature. Numerical simulations of Liu and Roble (2002) of the 2002 SSW event suggest that the extent of mesospheric cooling is between $60-110 \mathrm{~km}$, with a secondary warming between $110-170 \mathrm{~km}$. On the other hand, results of the 2002 SSW event simulated by Coy et al. (2005) show a rather confined mesospheric cooling layer which only extends to about $80 \mathrm{~km}$. Continuous monitoring of MLT temperatures from the ground provides the necessary observational evidence of the extent of mesospheric cooling needed to validate these modeling studies. In this paper, rotational temperatures derived from $\mathrm{OH}(6,2)$ and $\mathrm{O}_{2} \operatorname{At}(0,1)$ nightglow emissions from South Pole $\left(90^{\circ} \mathrm{S}\right)$ are analyzed to examine MLT temperature anomalies prior to the 2002 Southern Hemisphere SSW. This paper is organized as follows: We first describe the instrument used for $\mathrm{OH}$ and $\mathrm{O}_{2}$ airglow observations and the procedures for calculating rotational temperatures. We then present the results from the 2002 austral winter season when an unprecedented Southern Hemisphere SSW event was recorded (Baldwin et al., 2003). We conclude by summarizing the significant findings of this study.

\section{Instrumentation and temperature measurements}

Both $\mathrm{OH}$ Meinel $(6,2)$ and $\mathrm{O}_{2}$ At $(0,1)$ airglow measurements were made by a half meter focal length, modified Czerny-Turner CCD Spectrometer (CCDS) located at South Pole, Antarctica. It is fitted with a $50 \mathrm{~mm}$ arc length curved 

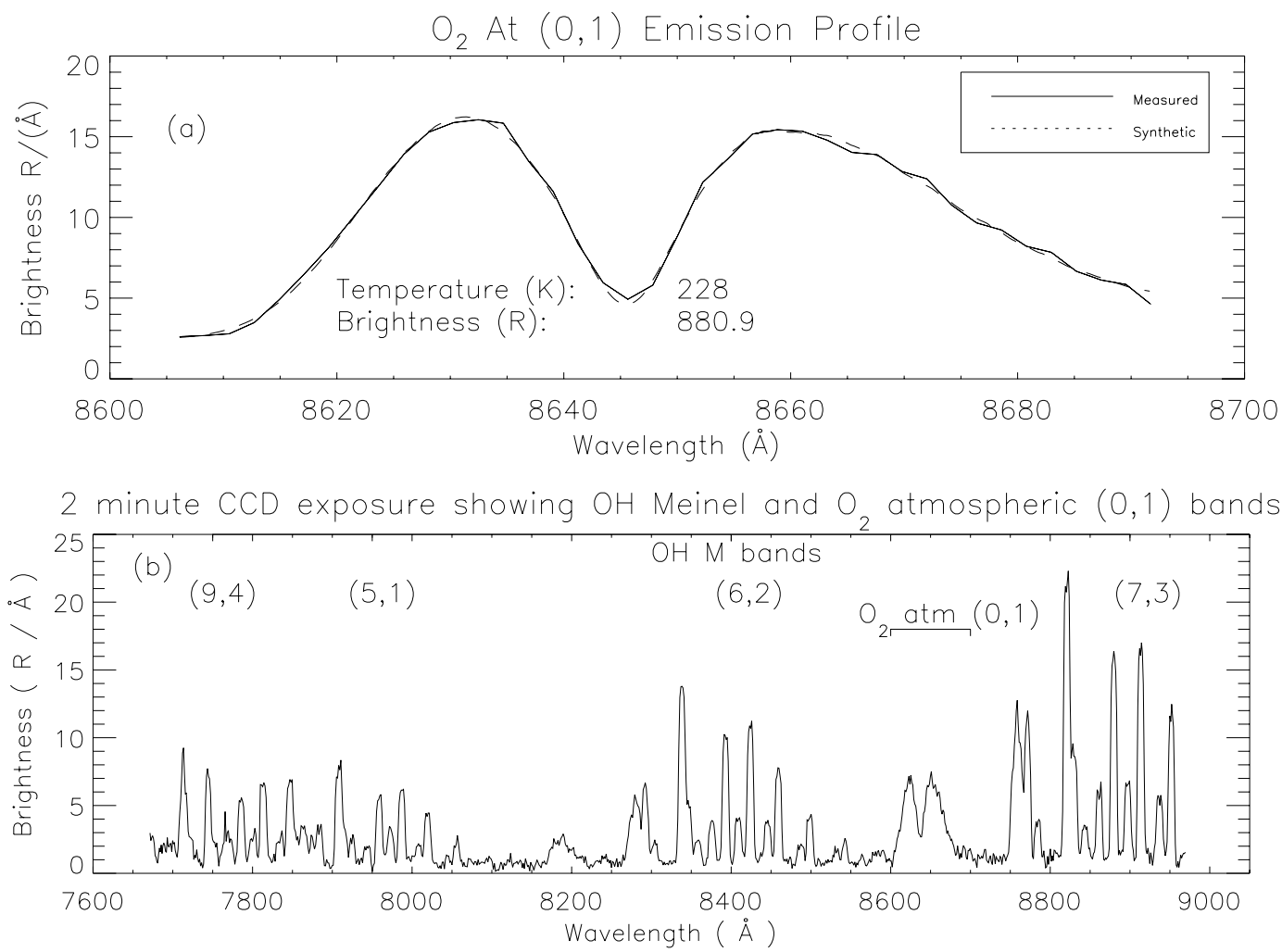

Fig. 2. (a) Measured and fitted $\mathrm{O}_{2} \mathrm{At}(0,1)$ band emission with (b) A typical 2-minuted averaged CCDS spectrum showing various nightglow features.

entrance slit and several serially replaceable 1200 groves $/ \mathrm{mm}$ grating; each grating is blazed at a different wavelength to maximize efficiency in the preferred spectral range and spectral order. Details of the instruments performance are given by Sivjee and Shen (1997). This CCDS operates continuously ( $24 \mathrm{~h}$ a day) for most of the six months of dark austral winter night from April to September. For the 2002 observing season, the instrument operated from 10 April to $28 \mathrm{Au}-$ gust and this study focuses on the data taken over the 1 June28 August 2002 period. The CCDS covers a free spectral of $\sim 1500 \AA$ in the near infra-red, has a circular field of view of $\sim 12^{\circ}$, and produces emission line profiles with an average full width half maximum (FWHM) of $\sim 7 \AA$. Exposure duration (spectral integration period) is approximately $2 \mathrm{~min}$. Kinetic temperatures in the MLT region are inferred from CCDS spectral measurements of $\mathrm{OH}(6,2)$ emission (mean altitude of $87 \mathrm{~km}$ ) and $\mathrm{O}_{2} \mathrm{At}(0,1)$ emission (mean altitude of $94 \mathrm{~km})$.

\subsection{OH temperature data}

Rotational population distribution temperatures were derived from the $\mathrm{OH}$ Meinel $(6,2)$ band using the procedure described in Sivjee and Hamwey (1987). Intensities of the rotational lines have been repeatedly shown to conform to a
Stefan-Boltzmann distribution of the excited $\mathrm{OH}$ molecules amongst the rotational levels of any vibrational state (Sivjee, 1992; Sivjee and Hamwey, 1987). This establishes the existence of thermal equilibrium between the $\mathrm{OH}$ and the ambient neutral atmosphere in the MLT region (Turnbull and Lowe, 1983). Thermalization results from the long radiative lifetime of the $\mathrm{OH}$ molecule during which each $\mathrm{OH}$ molecule collides on average about 10 times with the molecules of the ambient neutral atmosphere. The peak brightness of the $\mathrm{P}_{1}$ and $\mathrm{P}_{2}$ rotational lines (see Fig. 1a) can be expressed in terms of vibrational-rotational transition probabilities, $A(J)$ and rotational term values, $F(J)$ (Sivjee, 1992, and references therein).

$$
\begin{aligned}
I\left(v^{\prime}, v^{\prime \prime}, J^{\prime}, J^{\prime \prime}\right)= & \frac{1}{Q_{\mathrm{R}}} I_{o} 2\left(2 J^{\prime}+1\right) \\
& \cdot A\left(v^{\prime}, v^{\prime \prime}, J^{\prime}, J^{\prime \prime}\right) e^{\left(-F\left(v^{\prime}, J^{\prime}\right) h c / k T\right.}
\end{aligned}
$$

where $J$ is the total angular momentum, and $Q_{\mathrm{R}}$ is the rotational partition function. It follows that:

$\ln \left\{\frac{I\left(v^{\prime}, v^{\prime \prime}, J^{\prime}, J^{\prime \prime}\right)}{\left(2 J^{\prime}+1\right) A\left(v^{\prime}, v^{\prime \prime}, J^{\prime}, J^{\prime \prime}\right)}\right\}=$ constant $-\frac{F\left(v^{\prime}, J^{\prime}\right) h c}{k T}$

Performing a linear least squares fit of $\ln I /[(2 J+1) A]$ against $F(J)$ for the first six rotational lines yields a straight 

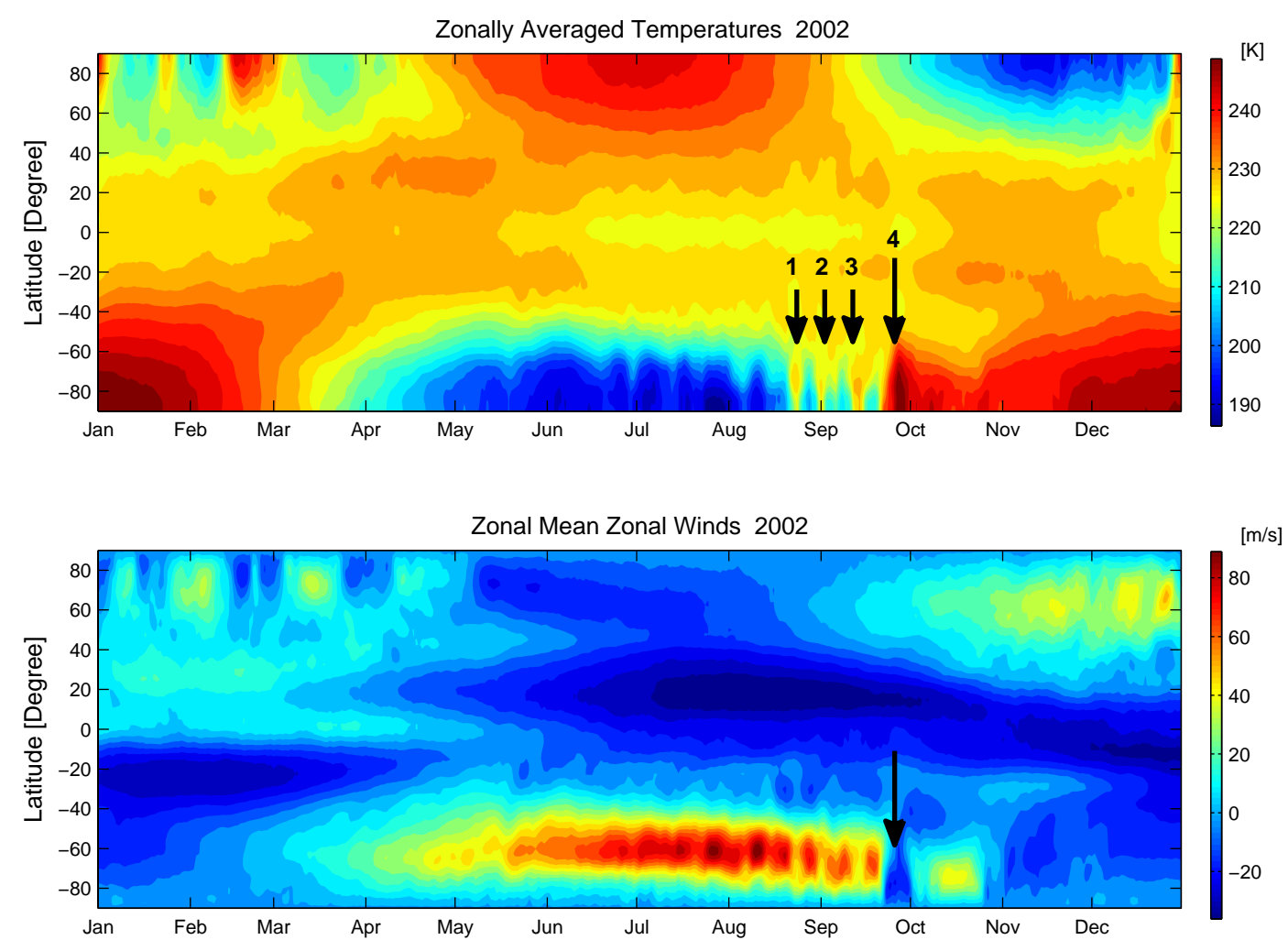

Fig. 3. Top panel: Zonally averaged temperatures from NCEP Reanalysis sampled at $36 \mathrm{hPa}$ shown as a function of latitude and universal time. Three minor warming and the major SSW event are marked by arrows. The first three minor warmings were centered on 24 August, 1 September, and 14 September while the major SSW event was centered on 27 September. Bottom panel: Latitude-time cross section of zonal mean zonal winds at $36 \mathrm{hPa}$ from NCEP Reanalysis showing the reversal of the wind at the onset of major SSW event.

line of slope $-h c / k T$ (see Fig. 1), from which the temperature $(T)$ can be easily calculated. Each spectrum and corresponding linear regression plot are individually analyzed to ensure that the derived temperatures are consistent with the thermalized population of the $\mathrm{OH}$ among the rotational states, and that there is no significant contamination due to bright lights or adverse viewing conditions.

\subsection{1 $\mathrm{O}_{2}$ temperatures}

$\mathrm{O}_{2} \operatorname{At}(0,1)$ rotational temperatures were calculated by convolving the synthetic (theoretical) emission profile of $\mathrm{O}_{2} \mathrm{At}$ band with the instrument function and fitting to measured spectral profiles as a function of temperature. In this study we have used purely airglow data free of any auroral contamination. The solid line in Fig. 2a shows a sample measured $\mathrm{O}_{2} \operatorname{At}(0,1)$ spectrum while the dashed line represent the corresponding synthetic fit. Figure $2 b$ shows a typical airglow spectrum recorded by the CCDS at the South Pole and used for deriving $\mathrm{O}_{2}$ rotational temperatures used in this study. $\mathrm{O}_{2}$ $\operatorname{At}(0,1)$ rotational temperatures and band brightness were estimated using the non-linear least square fitting technique of Yee et al. (1991).

\section{Results}

An unprecedented meteorological event associated with the SSW event was observed in the Southern Hemisphere during the winter of 2002. The Southern Hemisphere cold polar vortex in the stratosphere was disrupted in 2002, eventually breaking down and leading to a large-scale warming of the polar stratosphere over a few days period. Zonally averaged temperatures and zonal mean zonal winds from National Center for Environment Prediction (NCEP) Reanalysis dataset sampled at $10 \mathrm{hPa}$ are shown in Fig. 3 as a function of latitude and universal time. Figure 3 shows three minor warming events in the Southern Hemisphere stratosphere which occurred during late August and mid September. These events are marked in Fig. 3 (top panel) by short arrowheads labeled 1,2, and 3. During these events the zonal mean zonal wind in the Southern Hemisphere was weakened but did not produce a reversal in zonal-mean circulation (see Fig. 3 - bottom panel). A major SSW event in the Southern Hemisphere occurred in late September accompanied by a reversal in zonal mean zonal wind. The onset of the 2002 major SSW in the Southern Hemisphere is indicated in Fig. 3 by the large arrowheads. We define the onset of the SSW event as 

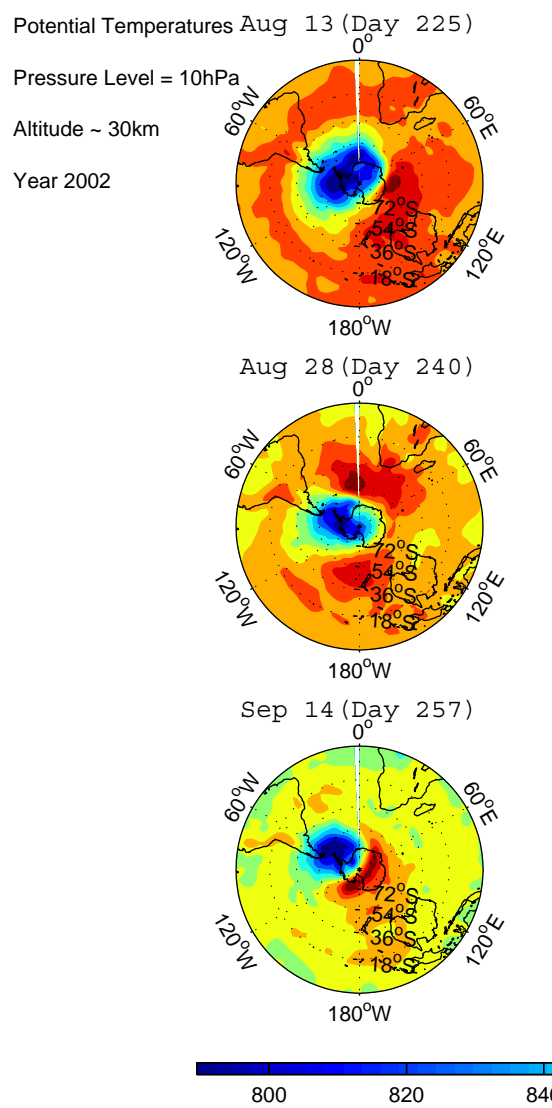
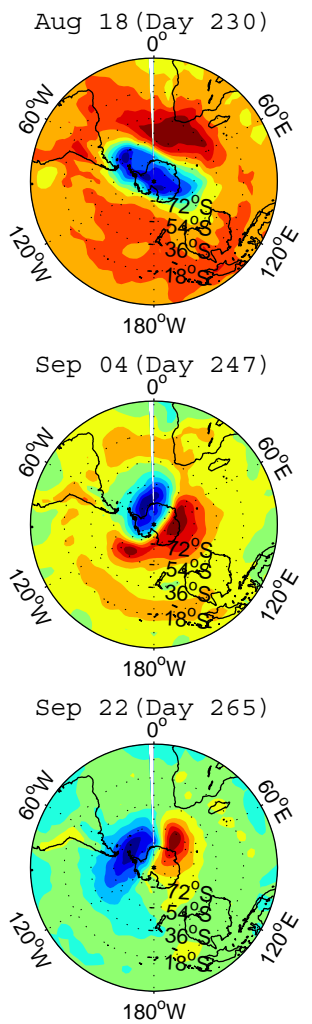
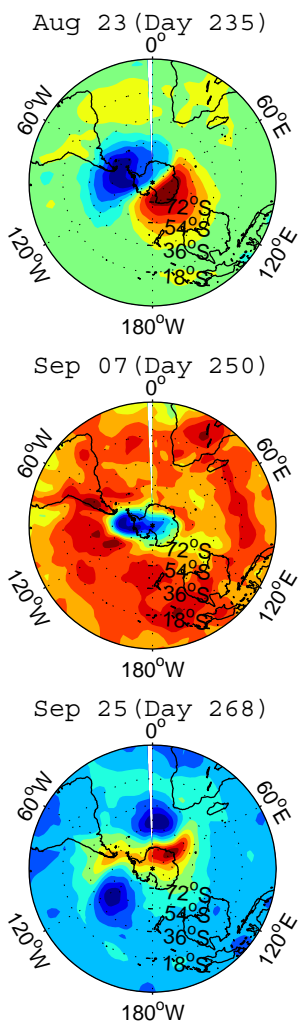

940 960

Fig. 4. Southern Hemisphere potential temperature maps at $10 \mathrm{hPa}$ pressure level for selected days in August and September of 2002. Fields are derived from NCEP temperatures.

the time of local peak in NCEP temperature ehancements at $10 \mathrm{hPa}$.

Figure 4 shows the Southern Hemisphere potential temperature maps at $10 \mathrm{hPa}[\sim 30 \mathrm{~km}]$ for selected days in $\mathrm{Au}-$ gust and September of 2002 leading up to the major SSW of late September 2002. At the start of the sequence shown in Fig. 4, the basic cyclonic polar vortex is well established and is centered just off the pole at $60^{\circ} \mathrm{W}$. Distortion of the polar vortex can be seen to commence on 18 August when the vortex (cyclone) becomes elongated and is slightly deformed by a warmer anticyclone (wave 1 warming). The whole pattern rotates eastward and on 23 August the anticyclone reaches its maximum amplitude accompanied by the first minor warming. Immediately following the first minor warming the anticyclone weakens and then peaks again on September 4 representing the second minor stratospheric warming (event 2 Fig. 3a). The polar vortex re-establishes itself on 7 September, but lasts for few days only, before being disrupted again by the warmer anticyclone on September 14 leading to the third minor warming. On 25 September the polar vortex splits into two cyclonic cells indicating the commencement of a wavenumber 2 stratospheric warming. This last warm- ing was also associated with the reversal of the zonal-mean zonal wind (see Fig. 3), corresponding to a major SSW event.

Variations of $\mathrm{OH}$ and $\mathrm{O}_{2}$ rotational temperatures at South Pole from June 2002 through August 2002 are shown in Fig. 5. The figure shows the behavior of MLT temperatures at South Pole leading up to the first minor warming of 2002 in the Southern Hemisphere. Temperatures shown in Fig. 5 have been smoothed using a 5-day running mean to filter out high frequency components. Vertical bars in the plot represent the estimated error in the mean. The mean value of $\mathrm{OH}$ temperature is about $212 \mathrm{~K}$ while $\mathrm{O}_{2}$ mean temperature is about $224 \mathrm{~K}$. For reference, the climatological mean of $\mathrm{OH}(3,1)$ rotational temperatures based on observations from 1994 through 2008 is also shown in Fig. 5. The details of $\mathrm{OH}(3,1)$ airglow measurements at the South Pole and the technique for estimating $\mathrm{OH}(3,1)$ rotational temperaturess are described in Azeem et al. (2005). Abrupt changes in $\mathrm{OH}$ and $\mathrm{O}_{2}$ temperatures can be seen in Fig. 5 centered on 20 July. Temperature anomalies seen in the observations show sudden cooling $(\Delta T=\sim 30 \mathrm{~K})$ in $\mathrm{OH}$ temperatures accompanied by warming $(\Delta T=\sim 15 \mathrm{~K})$ in $\mathrm{O}_{2}$ temperatures preceding the onset of the first minor SSW event. The cooling 


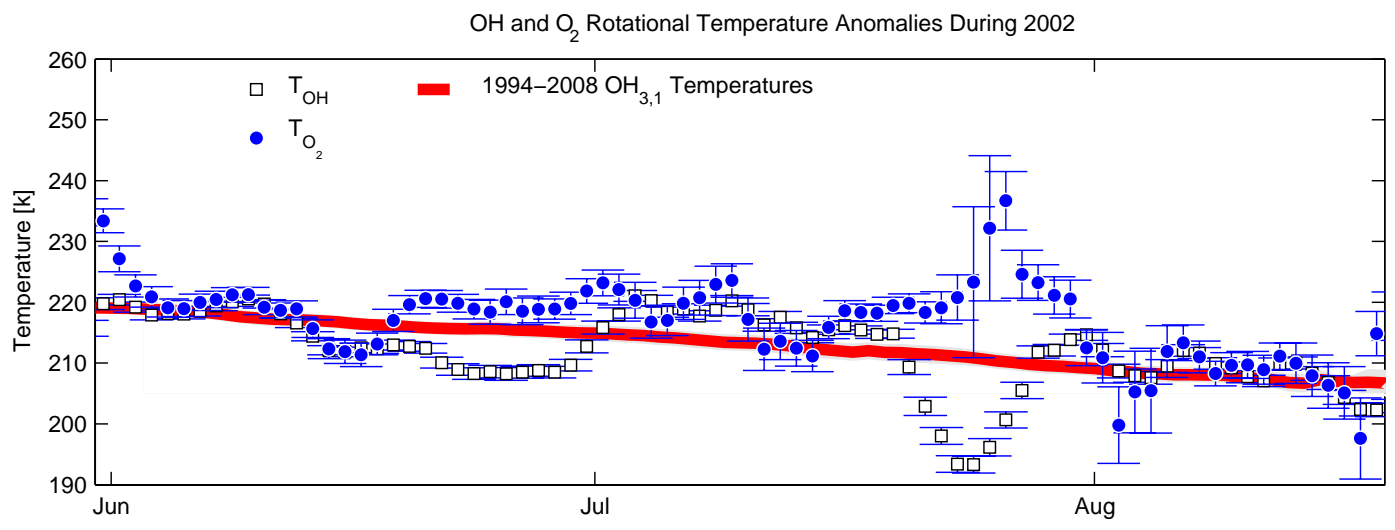

Fig. 5. 5-day smoothed $\mathrm{OH}$ (square) and $\mathrm{O}_{2}$ (filled circle) rotational temperatures at South Pole during 2002. The climatological mean of $\mathrm{OH}(3,1)$ rotational temperatures is shown in red for reference. Mesospheric cooling and lower thermospheric warming can be noted in $\mathrm{OH}$ and $\mathrm{O}_{2}$ rotational temperature data, respectively.

and warming trends seen in $\mathrm{OH}$ and $\mathrm{O}_{2}$ temperatures, respectively, suggest a much shallower extent of mesospheric cooling than reported by Liu and Roble (2002). Recent numerical simulations of the SSW event by Coy et al. (2005) also suggested a rather shallow mesospheric cooling layer though the vertical extent of the cooling in the model was confined to within the lower mesosphere.

To examine the presence and temporal evolution of longperiod waves in the MLT region before, during and after the onset of the stratospheric warming event, we Fourier analyzed the $\mathrm{OH}$ and $\mathrm{O}_{2}$ rotational temperature data for the period 1 June-31 August 2002. The scheme used to Fourier analyze the airglow temperature data is as follows: Each temperature dataset was hourly averaged and then a sinusoidal model together with a DC component was fitted to a block of data to determine the amplitudes and phases of harmonic components between 1 and 30 day period. The length of the data block was 2.5 times the period of the wave being fitted or at least 14 days. The block of data or window was then moved in time and the process was repeated to construct a spectrogram as shown in Fig. 6. The amplitude spectrograms of $\mathrm{O}_{2}$ and $\mathrm{OH}$ temperature data show strong planetary wave activity in the 5-10 day range. Comparing the timing of temperature anomalies (Fig. 5) and the evolution of planetary wave activity (Fig. 6), it can be seen that the maximum amplitude of $\mathrm{OH}$ and $\mathrm{O}_{2}$ vacillations and the peak in $\mathrm{OH}$ and $\mathrm{O}_{2}$ temperature anomalies occurred at the same time. The observed intensification of planetary wave activity in $\mathrm{OH}$ and $\mathrm{O}_{2}$ rotational temperatures at South Pole prior to the onset of SSW event is consistent with the results of Espy et al. (2005) and Pancheva et al. (2008) which also showed large planetary wave amplitudes in mesospheric wind measurements prior to the warming event.

To further examine the downward extent of the observed planetary wave enhancements, NCEP stratospheric tempera- ture field was analyzed for evidence of wave activity similar to that seen in $\mathrm{OH}$ and $\mathrm{O}_{2}$ temperature data. To quantify the wave characteristics (period and wavenumber) in the stratosphere at Southern polar latitudes during the SSW event, NCEP temperatures sampled at $85^{\circ} \mathrm{S}$ were Fourier analyzed. Figure 7 shows the longitude-time map of the detrended NCEP temperatures at $10 \mathrm{hPa}$ and the corresponding power spectrum of the perturbations in the temperature field. The results indicate that the dominant dynamical feature in the stratosphere during this period is the 10-day, zonal wavenumber 1 (Z1) planetary wave. It is worth noting that similar wave periods were also seen in the $\mathrm{OH}$ and $\mathrm{O}_{2}$ wavelet spectra at the peak of observed temperature anomalies. The presence of the 10-day wave in the MLT and in the stratosphere is suggestive of the vertical propagation of the wave during the SSW event. It is well known that SSW events are associated with the amplification of planetary waves in the stratosphere (Baldwin et al., 2003). The daily amplitudes of Z1 wave in NCEP temperatures averaged over the latitudes between $70^{\circ} \mathrm{S}$ and $85^{\circ} \mathrm{S}$ at $10 \mathrm{hPa}$ are shown in Fig. 8. We note that during the months of August and September wave events with large daily Z1 amplitudes are present. Baldwin et al. (2003) have shown that these amplitudes in 2002 were the largest amplitudes of wave 1 ever recorded in the Southern Hemisphere and were associated with the 2002 SSW event. Spectral analyses of the NCEP and MLT airglow temperature data reveal a number of features suggestive of the vertical linkage between the stratosphere and the MLT region during SSW events. First, the amplification of Z1 amplitude in NCEP temperature data during the Austral winter of 2002 is associated with the 2002 SSW event in the Southern Hemisphere. This Z1 planetary wave has a periodicity 10 days in the stratosphere and the same periodicity also appears in the $\mathrm{OH}$ and $\mathrm{O}_{2}$ airglow temperature spectra. Furthermore, the amplitude of the MLT planetary waves 

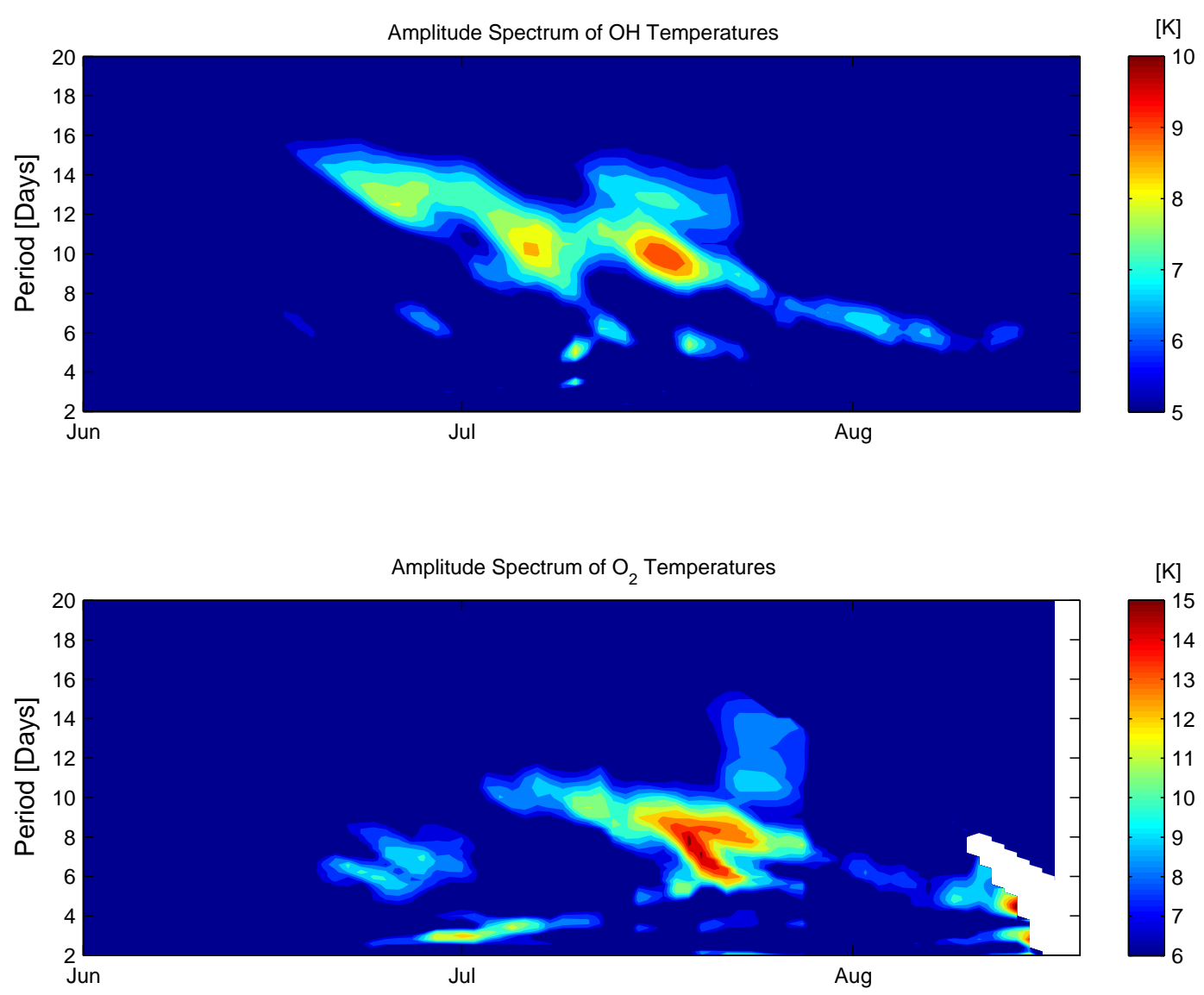

Fig. 6. Wavelet amplitude spectra of $\mathrm{OH}$ temperatures (top) and $\mathrm{O}_{2}$ temperatures (bottom) showing amplification of 5-10 day wave activity prior to the onset of SSW and coinciding with the MLT temperature anomalies seen in Fig. 5.

appears to be correlated with $\mathrm{OH}$ and $\mathrm{O}_{2}$ temperature anomalies. Given the suggestion in the data of the vertical propagation of the 10-day Z1 wave associated with the SSW event, we examine the association between $\mathrm{Z} 1$ wave amplitudes and MLT temperature anomalies. Figure 9 shows the crosscorrelation between $\mathrm{Z} 1$ amplitudes and airglow temperatures (from Fig. 5) over 1 July-31 August 2002 period. OH rotational temperatures are anti-correlated with $\mathrm{Z} 1$ amplitudes and appears to lag behind the $\mathrm{Z} 1$ amplitudes by 4 days while $\mathrm{O}_{2}$ rotational temperatures show positive correlation with $\mathrm{Z1}$ amplitudes with a 7-day lag. Since the analysis of NCEP stratospheric temperatures during SSW events showed 10day zonal wavenumber 1 to be the dominant feature, we have only included $\mathrm{Z} 1$ amplitudes in this cross-correlation analysis. The presence of $Z 1$ wave in the stratosphere and MLT regions and the observed correlation between $\mathrm{Z} 1 \mathrm{am}-$ plitudes and airglow temperature anomalies suggests that the observed changes in MLT temperature are precursors to the SSW event.

To explain the delay between observing MLT temperature anomalies and the onset of SSW event, which in this case was about 3-4 weeks, we look at the cross-correlation be- tween daily Z1 amplitudes (see Fig. 8) and NCEP temperatures sampled at $10 \mathrm{hPa}$ and averaged over the latitudes between $70^{\circ} \mathrm{S}$ and $85^{\circ} \mathrm{S}$ for the period 1 July-15 October 2002. The cross-correlation shows that $\mathrm{Z} 1$ amplitudes lead temperature changes in the stratosphere by about 30 days. It should be noted here that stratospheric temperatures over this periods were characterized by 3 minor SSW events followed by the major event in September and thus this cross-correlation represents quantitatively the association between the planetary waves and SSW events. It was shown earlier that the MLT temperature anomalies in $\mathrm{OH}$ and $\mathrm{O}_{2}$ rotational temperature data lag behind $\mathrm{Z} 1$ amplitudes by 3-7 days. When this lag is taken into account, one can see that the observed MLT temperature anomalies are expected to lead the SSW event by 3-4 weeks as observed.

\section{Summary and conclusions}

A major stratospheric warming event was observed in 2002 in the Southern Hemisphere. The major warming was preceded by a sequence of three minor warmings. In this paper 

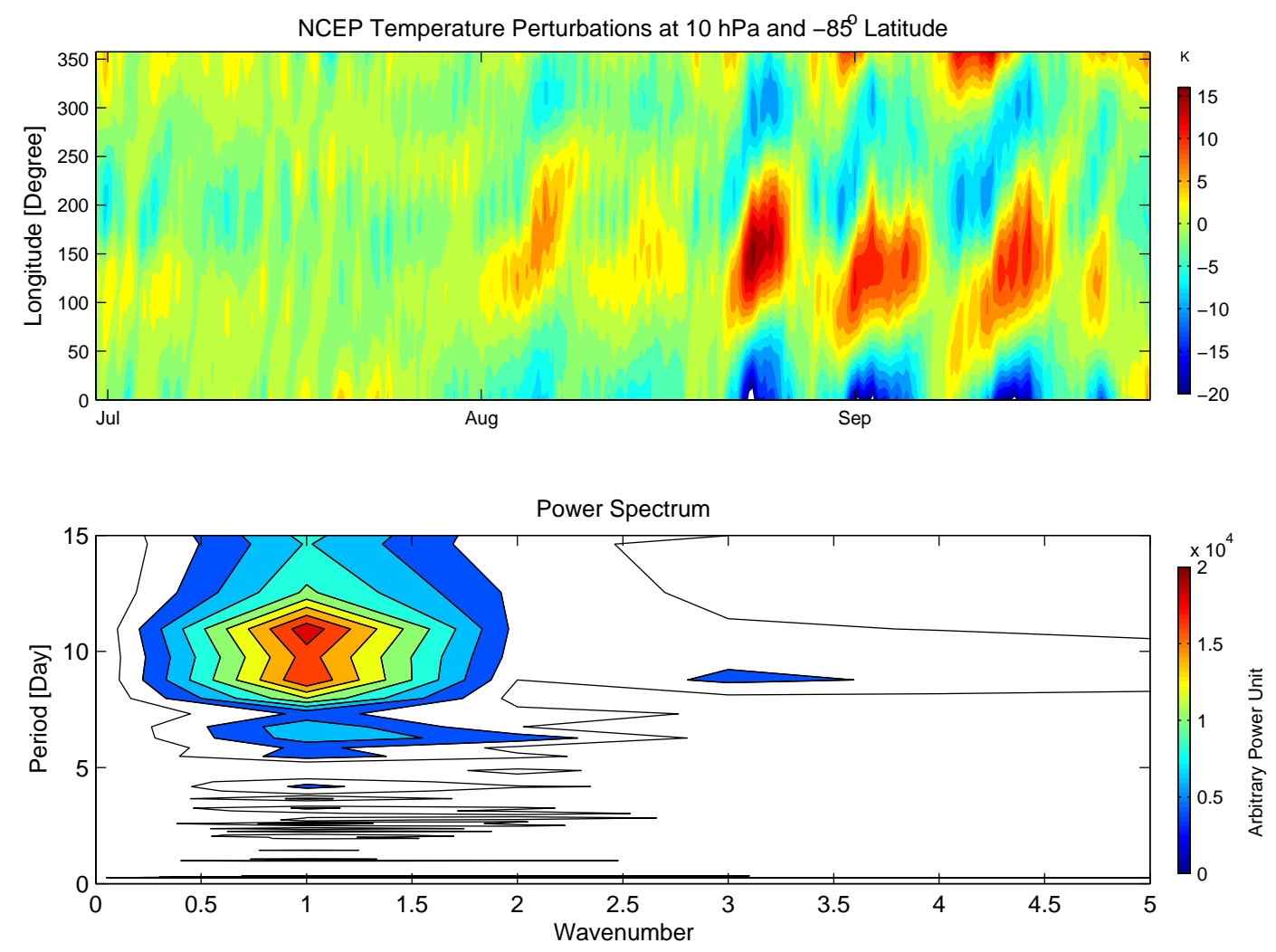

Fig. 7. Top: Longitude-time map of NCEP temperatures at $10 \mathrm{hPa}$ and at $85^{\circ} \mathrm{S}$ latitude. Bottom: Power spectrum of the NCEP temperatures shown in the top panel showing the presence of a strong zonal waveumber 1 and 10-day fluctuation in the data.

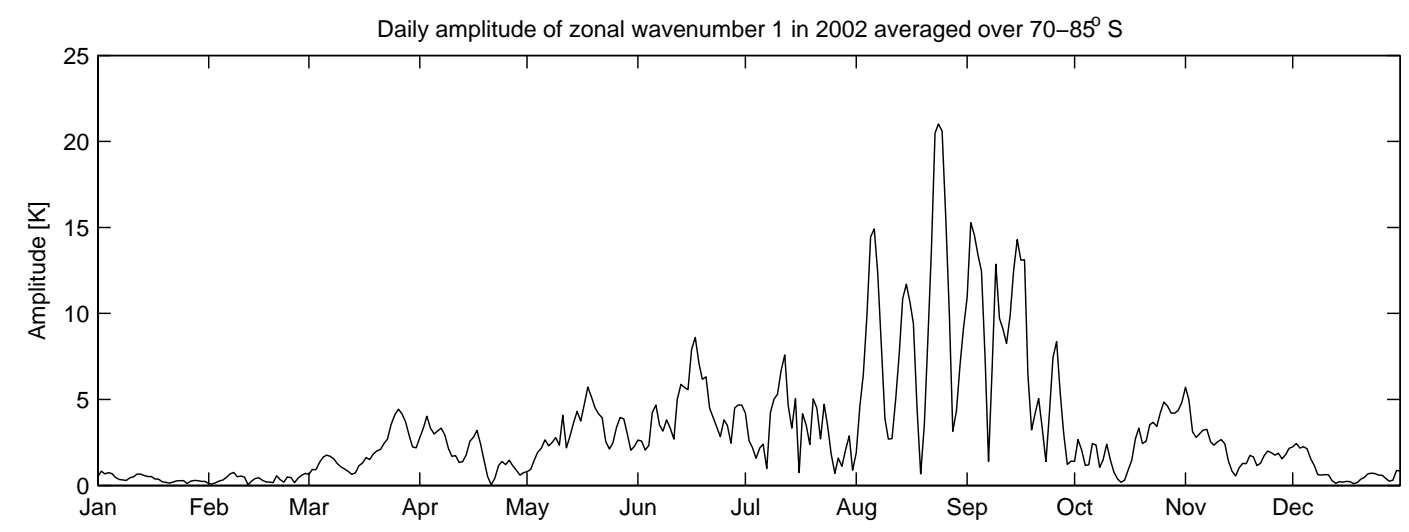

Fig. 8. Daily amplitudes of zonal waveumber 1 (Z1) computed form NCEP temperatures averaged over the latitudes between $70^{\circ} \mathrm{S}$ and $85^{\circ} \mathrm{S}$ and at the $10 \mathrm{hPa}$ level.

we have analyzed airglow temperature data from the South Pole during the 2002 austral winter to examine large temperature anomalies in the MLT region associated with the SSW. Kinetic temperature at $\sim 87$ and $\sim 94 \mathrm{~km}$ inferred from $\mathrm{OH}(6,2)$ and $\mathrm{O}_{2} \mathrm{At}(0,1)$ airglow measurements, respectively, show abrupt temperature changes in the MLT region occurring three to four weeks prior to the onset of wave 1 warming in the stratosphere. Temperature anomalies observed in the MLT region show sudden cooling $(\Delta T=\sim 30 \mathrm{~K})$ in $\mathrm{OH}$ temperatures accompanied by warming $(\Delta T=\sim 15 \mathrm{~K})$ in $\mathrm{O}_{2}$ temperatures. The dynamical response of the MLT region to these large temperature anomalies was assessed by examining the behavior of the planetary wave activity seen in the airglow temperature dataset. Wave spectra of $\mathrm{OH}$ and $\mathrm{O}_{2}$ airglow temperatures show amplification of 5-10 day waves prior to the onset of SSW. The timing of wave amplification 

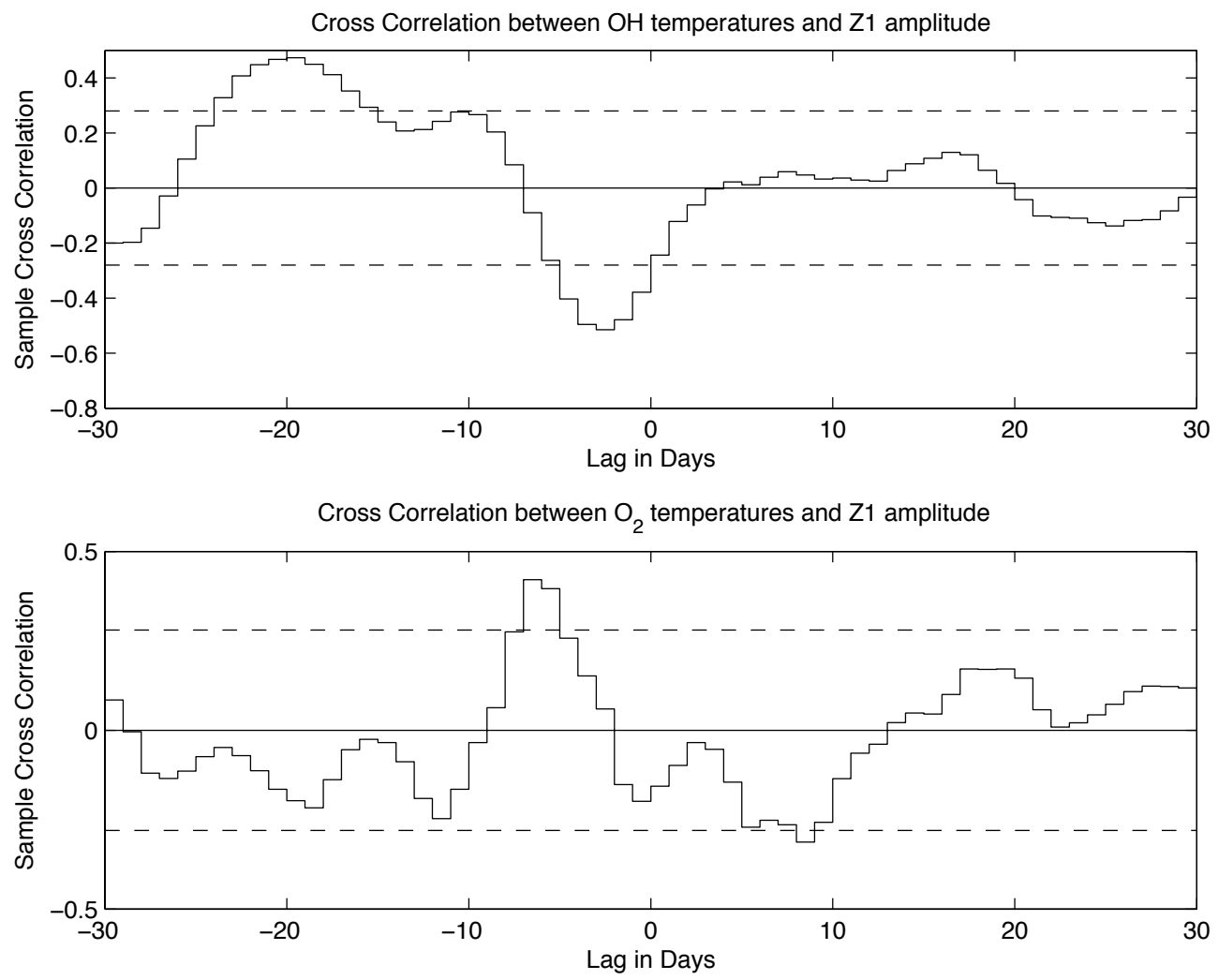

Fig. 9. Top: Cross-correlation between $\mathrm{OH}$ rotational temperatures (shown in Fig. 5) and daily temperature amplitudes of $\mathrm{Z1}$ (shown in Fig. 8). Negative lag here represents $\mathrm{OH}$ temperatures lagging $\mathrm{Z} 1$ amplitudes. Dashed lines are the upper and lower $95 \%$ confidence bounds. Bottom: Same as the top panel but for $\mathrm{O}_{2}$ rotational temperatures.

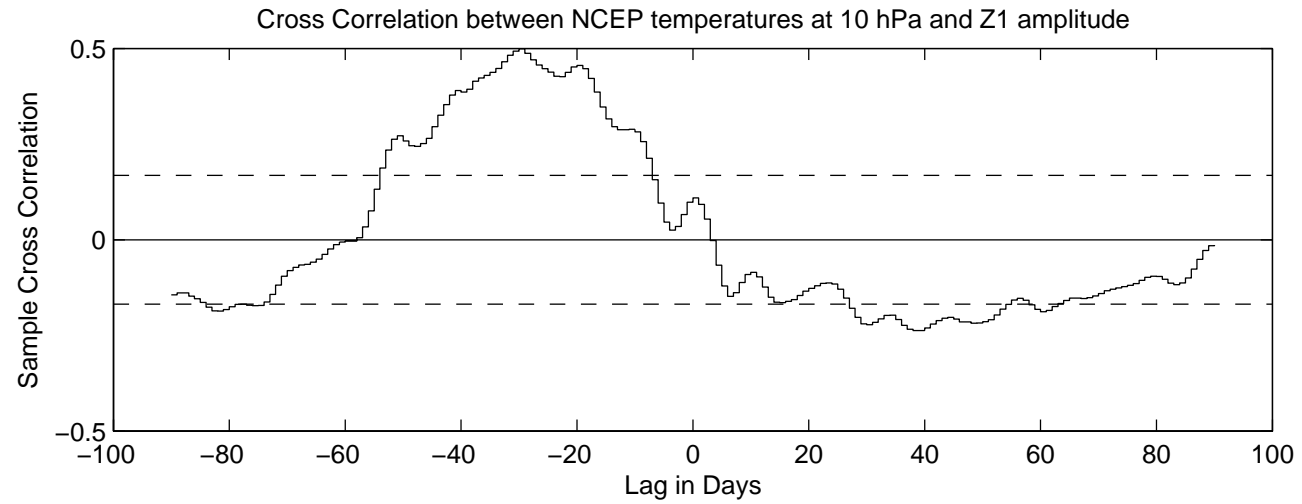

Fig. 10. Cross-correlation between NCEP temperatures at $10 \mathrm{hPa}$ and daily $\mathrm{Z} 1$ amplitudes. Both the NCEP temperatures and Z1 amplitudes were averaged over the latitudes between $70^{\circ} \mathrm{S}$ and $85^{\circ} \mathrm{S}$. Dashed lines are the upper and lower $95 \%$ confidence bounds.

seen in the wave spectra coincides with the peak in $\mathrm{OH}$ and $\mathrm{O}_{2}$ temperature anomalies.

In this paper we have presented airglow temperatures from $\mathrm{OH}$ Meinel $(6,2)$ and $\mathrm{O}_{2} \operatorname{At}(0,1)$ band emissions showing the vertical extent of the mesospheric cooling which precedes SSW events. Such observations of the extent of mesospheric cooling are needed to validate various modeling stud- ies of SSW events and to provide a mechanism to constrain gravity wave parameterization and planetary wave forcing prescribed in the models. There are two possibilities concerning the observed temperature anomalies preceding the minor SSW events. According to Liu and Roble (2002), changes in the penetration regime of the gravity waves during the SSW can drive meridional circulation leading to adiabatic 
cooling and heating in the MLT region. Alternately, a second mechanism of these temperature changes proposed by Thayer and Livingston (2008) suggests that regional baroclinic zones can drive circulation leading to adiabatic cooling in the mesosphere. Better understanding of the source and timing of temperature anomalies in the MLT region associated with SSW events would require further analysis of the divergence of Q-vector near baroclinic zones (Thayer and Livingston, 2008) and Eliassen-Palm flux divergence diagnostic in the MLT temperatures (Zhu et al., 2008).

Acknowledgements. This work was supported by the National Science Foundation grants OPP-03352742 and ATM-0449864 to Embry-Riddle Aeronautical University. We acknowledge the use of NCEP Reanalysis data provided by the NOAA/OAR/ESRL PSD, Boulder, Colorado, USA, from their Web site at http://www. esrl.noaa.gov/psd/. ERT and JHY were supported by NSF grant ATM 0640864.

Topical Editor C. Jacobi thanks two anonymous referees for their help in evaluating this paper.

\section{References}

Azeem, S. M. I., Talaat, E. R., Sivjee G. G., Liu, H.-L., and Roble, R. G.: Observational study of the 4-day wave in the mesosphere preceding the sudden stratospheric warming events during 1995 and 2002, Geophys. Res. Lett., 32, L15804, doi:10.1029/2005GL023393, 2005.

Baldwin, M, Hirooka, T., O’Neill, A., and Yoden, S.: Major stratospheric warming in the Southern Hemisphere in 2002: Dynamical aspects of the ozone hole split, SPARC Newsletter 20, 2003.

Cho, Y.-M., Shepherd, G. G., Won, Y.-I., Sargoytchev, S., Brown, S., and Solheim, B.: MLT cooling during stratospheric warming events, Geophys. Res. Lett., 31, L10104, doi:10.1029/2004GL01955, 2004.

Coy, L., Siskind, D. E., Eckermann, S. D., McCormack, J. P., Allen, D. R., and Hogan, T. F.: Modeling the August 2002 minor warming event, Geophys. Res. Lett., 32, L07808, doi:10.1029/2005GL022400, 2005.

Dowdy , A. J., Vincent, R. A., Tsutsumi, M., Igarashi, K., Murayama, Y., Singer, W., Murphy, D. J., and Riggin, D. M.: Polar mesosphere and lower thermosphere dynamics: 2. Response to sudden stratospheric warmings, J. Geophys. Res., 112, D17105, doi:10.1029/2006JD008127, 2007.

Espy, P. J., Hibbins, R. E., Riggin, D. M., and Fritts, D. C.: Mesospheric planetary waves over Antarctica during 2002, Geophys. Res. Lett., 32, L21804, doi:10.1029/2005GL023886, 2005.

Hernandez, G.: Winter mesospheric temperature above South Pole $\left(90^{\circ} \mathrm{S}\right)$ and their relationship to the springtime ozone hole size, Geophys. Res. Lett., 31, L07109, doi:10.1029/2004GL019414, 2004.

Hernandez, G.: Climatology of the upper mesosphere temperature above South Pole (90oS); Mesospheric cooling during 2002, Geophys. Res. Lett., 30(10), 1535, doi:10.1029/2003GL016887, 2003.

Labitzke, K.: On the mutual relation between stratosphere and troposphere during periods of stratospheric warmings in winter, J. Appl., Meteorol, 4, 91-99, 1965.
Labitzke, K. and Naujokat, B.: The lower arctic stratosphere midwinter warmings since 1952, SPARC Newsletter, 15, 11-14, 2000.

Lauter, E. A. and Schminder, R.: Pressure and wind variations in the mesopause region, resulting from stratospheric warmings, Physica Solariterrestris, 1, 111-119, 1976.

Liu, H.-L. and Roble, R. G.: A study of a self-generated stratospheric sudden warming and its mesospheric-lower thermospheric impacts using the coupled TIMEGCM/CCM3, J. Geophys. Res., 107, 4695-4712, 2002.

Lysenko I. A., Portnyagin, Yu. I., Greisigerm, K. M., and Sprenger, K.: Some peculiarities of the atmospheric circulation at the altitude of 90-100 km over Europe in winter 1972-73, J. Meteorol., 25, 213-217, 1975.

Mbatha, N., Sivakumar, V., Malinga, S. B., Bencherif, H., and Pillay, S. R.: Study on the impact of sudden stratosphere warming in the upper mesosphere-lower thermosphere regions using satellite and HF radar measurements, Atmos. Chem. Phys. Discuss., 9, 23051-23072, 2009, http://www.atmos-chem-phys-discuss.net/9/23051/2009/.

Mukhtarov, P., Pancheva, D., Andonov, B., Mitchell, N. J., Merzlyakov, E., Singer, W., Hocking, W., Meek, C., Manson, A., and Murayama, Y.: Large-scale thermodynamics of the stratosphere and mesosphere during the major stratospheric warming in 2003/2004, J. Atmos. Sol.-Terr. Phys., 69, 2338-2354, 2007.

Myrabø, H. K., Deehr, C. S., and Lybekk, B.: Polar cap OH airglow rotational temperatures at the mesopause during a stratospheric warming event, Planet. Space Sci., 32, 853-856, 1984.

Pancheva, D., Mukhtarov, P., Mitchell, N. J., Andonov, B., Merzlyakov, E., Singer, W., Murayama, Y., Kawamura, S., Xiong, J., Wan, W., Hocking, W., Fritts, D., Riggin, D., Meek, C., and Manson, A.: Latitudinal wave coupling of the stratosphere and mesosphere during the major stratospheric warming in 2003/2004, Ann. Geophys., 26, 467-483, 2008, http://www.ann-geophys.net/26/467/2008/.

Scherhag, R.: Stratospheric temperature changes and the associated changes in pressure distribution, J. Meteorol., 17, 572-585, 1960.

Schoeberl, M. R.: Stratospheric warmings: observations and theory, Rev. Geophys. Space Phys., 16, 521-537, 1978.

Sivjee, G. G. and Shen, D.: Auroral optical emissions during the solar magnetic cloud event of October 1995, J. Geophys. Res., 102, 7431-7437, 1997.

Thayer, J. P. and Livingston, J. M.: Observations of wintertime arctic mesosphere cooling associated with stratosphere baroclinic zones, Geophys. Res. Lett., 35, L18803, doi:10.1029/2008GL034955, 2008.

Yee, J-H., Niciejewski, R., and Luo, M. Z.: Observations of $\mathrm{O}_{2}\left({ }^{1} \Sigma\right)$ and $\mathrm{OH}$ nightglow during the ALOHA-90 campaign, Geophys. Res. Lett., 18, 1357-1360, 1991.

Walterscheid, R. L., Sivjee, G. G., and Roble, R. G.: Mesospheric and lower thermospheric manifestations of a stratospheric warming event over Eureka, Canada $\left(80^{\circ} \mathrm{N}\right)$, Geophys. Res. Lett., 27, 2897-2900, 2000.

Zhu, X., Yee, J.-H., Talaat, E. R., Mlynczak, M., and Russell III, J. M.: Diagnostic Analysis of Tidal Winds and the Eliassen-Palm Flux Divergence in the Mesosphere and Lower Thermosphere from TIMED/SABER Temperatures, J. Atmos. Sci., 65, 38403859, 2008. 